\title{
Application of Deep Learning Approaches in Igneous Rock Hyperspectral Imaging
}

\author{
Brian Bino Sinaice ${ }^{1(\&)}$, Youhei Kawamura ${ }^{1}$, Jaewon Kim ${ }^{1}$, \\ Natsuo Okada ${ }^{2}$, Itaru Kitahara ${ }^{3}$, and Hyongdoo Jang ${ }^{4}$ \\ Graduate School of International Resource Sciences, Akita University, \\ 1-1 Tegatagakuenmachi, Akita, Akita Prefecture 010-8502, Japan bsinaice@ rocketmail.com \\ 2 \\ Faculty of International Resource Sciences, Akita University, \\ 1-1 Tegatagakuenmachi, Akita, Akita Prefecture 010-8502, Japan \\ Center for Computational Sciences, University of Tsukuba, \\ 1-1-1 Tennodai, Tsukuba, Ibaraki 305-8577, Japan \\ Western Australian School of Mines Minerals, Energy and Chemical Engineering, Curtin University, Kalgoorlie, \\ WA 6430, Australia
}

\begin{abstract}
Hyperspectral imaging has been applied in remote sensing amongst other disciplines, success in these has triggered its extensive use. Hence, it comes as no surprise that we took advantage of this technology by conducting a study aimed at the spectral analysis of several igneous rocks, and to deduce the spectral signatures of each rock unit using neural networks. Through visual observations and comparisons of these spectral signatures, parameters such as band curvature(shape), tilt(position) and strength were used for lithological discrimination. Even with this said, there often exists similarities in rocks, which are rather difficult to differentiate by means of visual or graphical analysis. However, with numerous technologies making new waves in today's era and artificial intelligence (AI) being at the forefront of these developments, it was best fitting to employ deep learning, often referred to as a subset of AI; to train/learn from these hyperspectral signatures with a goal aimed at classifying these rocks. Deep learning has networks such as the convolution neural network $(\mathrm{CNN})$, which has algorithms that excel in feature representation from visual imagery; taking into account that the more data is fed into the training process and later used as a database for further training, the higher the future prediction accuracy. Gathered outcomes from the CNN show exceptionally high prediction accuracy capabilities of $96 \%$; suggesting viable field and laboratory usage of these systems as a unit for mining and rock engineering applications.
\end{abstract}

Keywords: Deep learning Hyperspectral imaging Convolution neural network

\section{Introduction}

Light as we see it via our human eyes does not let us detect material information at wavelengths beyond the visible light range (Tompkins and Pieters 1999), it is for this reason that this paper employs the use of wavelength ranges from 380-1000 nm, usually referred to by the science community as the 'Visible-Near-Infrared (VNIR)' range, as it gives out more information due to its spectral range (Pieters et al. 2009). Another aspect that comes into action is spectral resolution (Kruse 2012), this is the number of spectral bands (125 for this study) present within a given spectral range. Simply put, hyperspectral imaging is the collection of image spectra from 100s of spectral bands.

Dealing with this much data requires artificial intelligence (AI), under AI, machine learning comes into play, a subset of this machine learning is deep learning, this has networks such as the convolution neural network(CNN) employed for this study; which has proven able to learn from multitudes of imagery data (Xing et al. 2017), keep it, and reemploy whatever leant as a database for times in which the CNN comes across similar or unrelated data types (Poria et al. 2016), in this case, hyperspectral signatures of igneous rocks.

For industrial viability and economically sound mineral production, quick and accurate rock classification is significantly important; to achieve this, hyperspectral imaging of rocks can be used to differentiate rock units. To study these technologies and how effective they are, it requires the use of closely related rocks. A total of 8 igneous rock units were used, 4 plutonic rocks and 4 of their equivalent volcanic counterparts. Capturing and visualization of their hyperspectral images, classification through deep learning techniques and the subsequent prediction 
accuracy score (Jia et al. 2016) of these combined technologies are suggested as a vital tool to be employed in current and future mining activities seeing as that the world is moving into a technology and highly computer oriented phase, such as shown in Fig. 1.

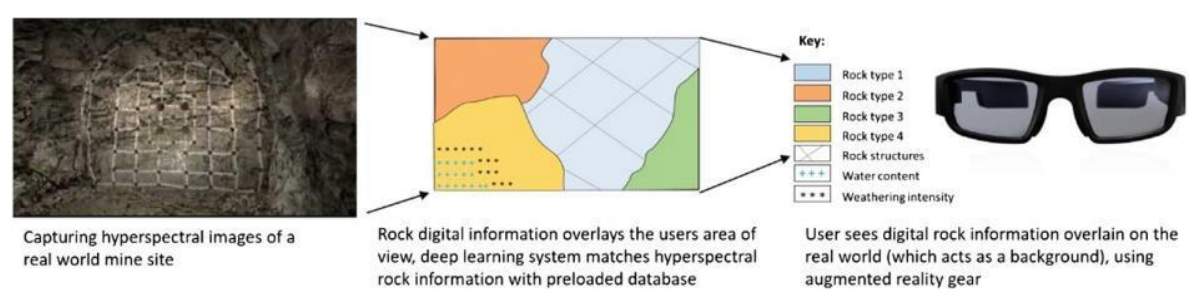

Fig. 1. How hyperspectral imaging and deep learning could be employed in future mining; combined with augmented reality, this system could revolutionize the mining industry.

\section{Background}

Arial compositional mapping of locations based on water content, lithology, soil and vegetation varieties (Meer 2006) are very important aspects of what helps scientists understand and at times predict what has or will take place from location to location, hence find ways to best engineer such localities. This requires deep understanding, detection and correct classification of rocks.

\section{$2.1 \quad$ Hyperspectral Imaging}

It is known that the regions beyond the visible light portions provide the most diagnostic absorption features (Kruse 2012), combined with a high spectral resolution, the collective spectral range (380-1000 nm) forms a robust, highly distinguishing method to identify and illustration spectral signature differences between rocks. The huge advantage of employing high resolution spectral methods is in its ability to draw data from an image input on a pixel-by-pixel basis from hundreds of narrow, adjacent spectral bands, thereby allowing thorough extraction of data (Pieters et al. 2009).

Spectral reflectance, which is the backbone of spectral signatures, varies from material to material due to the degree of energy differences along a given wavelength range (Meer 2006). Also referred to as the ratio of reflected energy to incident energy as a function of wavelength (Kruse 2012). Spectral bands where the signatures show a prominent downward deflection are said to be absorption bands of that particular material, thereby making it easy for material discrimination.

\subsection{Deep Learning}

There are a lot of proposed methods out there aimed at image data recognition, amongst these, deep learning has been at the forefront of current developments due to its reliability and near perfect recognition capabilities relative to the rest. A great deal of this capability comes from its ' $\mathrm{CNN}$ '.

Xing et al. (2017), point out that different granularities can be learnt and discriminated by CNN models directly from data, this gives them an advantage when dealing with multitudes of problems at the same time. Jia et al. (2016), also point out that higher-level structural patterns in features learned at the last layers are what CNNs models are said to implicitly capture. In the following text, we focus our discussion on 8 igneous rock unit datasets, we inspect each image and preform data pre-processing afore inputting rock spectral data for learning. This was done in two ways, first, splitting plutonic and volcanic rocks, thereafter importing and classifying each rock separately into the 8 igneous rock units employed for this study. Poria et al. (2016), further point out that from images collected, at times wide variations or similarities which may not be visually obvious to the human eye are effortlessly pinpointed by CNNs. As pointed out by authors such as Simonyan et al. (2013), the model architecture is also very important for the deep rock classification problem, hence models are continuously developed for various studies in which they are intended for application.

\section{Methodology}


Spectral techniques have become famous in the compositional differentiation of rocks, like any other science discipline where diagrams are used to represent a phenomenon, hyperspectral signature shapes depend of three main variables. Tompkins and Pieters (1999); Pieters et al. (2009) mention that the first variable; band tilt, shows the band absorption sensitivity. The second variable; band strength, shows the intensity of light being reflected. The third variable; band curvature, is a representation of how light is affected by a few adjacent and connected bands such that a wavy amplitude or trough across that section becomes evident (Kruse 2012). All these are based upon a 'white reference' which creates a straight line anomaly or standard from which all subsequent data emanates.

\subsection{Capturing the Hyperspectral Image}

8 igneous rock units from Akita mining museum were employed for this experiment, known beforehand and named accordingly before data collection.

To carry out pixel by pixel hyperspectral data collection from the 8 igneous rock units in question, an experimental setup was put in place, this consisted of a hyperspectral camera (380-1000 nm, 125 bands), a studiolike stage and a computer to operate the whole undertaking as shown in Fig. 2. Capturing the hyperspectral images entails placing a white reference board on the illuminated stage and capturing its hyperspectral image, hence creating the above mentioned background reference (Meer 2006). With that done, the board is replaced with a rock sample of the rock in question and its hyperspectral image captured.

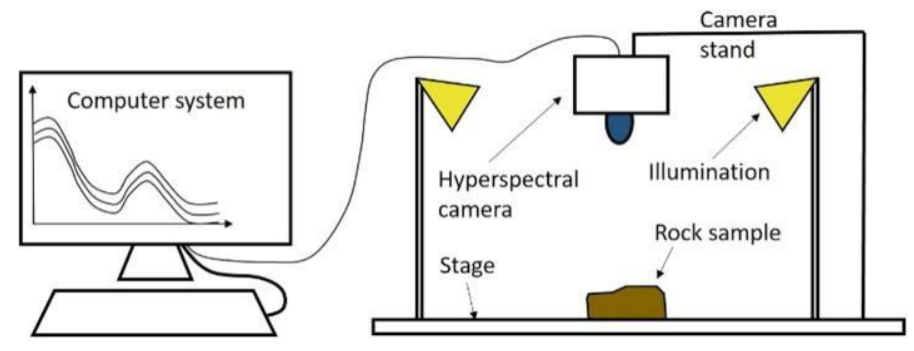

Fig. 2. Main components of a hyperspectral imaging experimental setup.

\subsection{Pixel Data Collection}

The analogue data captured by the camera requires some conversions before the digital analysis can begin, this entails the use of a hyperspectral analyzing software. Figure 3 shows the manner in which this is done by allowing the user to select how much area of the captured image they would like to employ for analysis, by selecting multiple smaller sized image subsets of the whole image so as to gather spectral information in fine detail. This creates hundreds of anomalies from smaller-sized subsets of the entire image, hence each anomaly representing each image partition's interactions with light across the spectral range. From this data, we can re-manipulate it to get hyperspectral signatures of each rock.

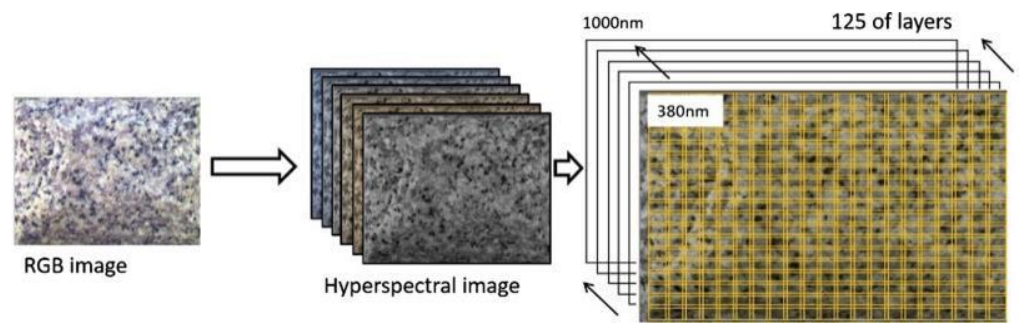

Fig. 3. Image splitting into smaller portions in order get representative data of each small area from the whole hyperspectral image. The RGB image has less spectral resolution (1 layer) compared to the hyperspectral image with 125 spectral channels.

\subsection{Data Pre-processing}

Spectral signatures from the hundreds of individual subsets of each rock image provide enough data for machine learning to take place. However, Simonyan et al. (2013), state that data pre-processing provides the best solution by adding or subtracting data through over and under-sampling to balance the underlying data, hence this process 
(Fig. 4). Numerous other data scientists such as Jia et al. (2016), vouch $80 \%$ learning, $10 \%$ validation and $10 \%$ testing as the ultimate ratio to yield the best output. The CNN is set to automatically and randomly split the complete dataset into these ratios.

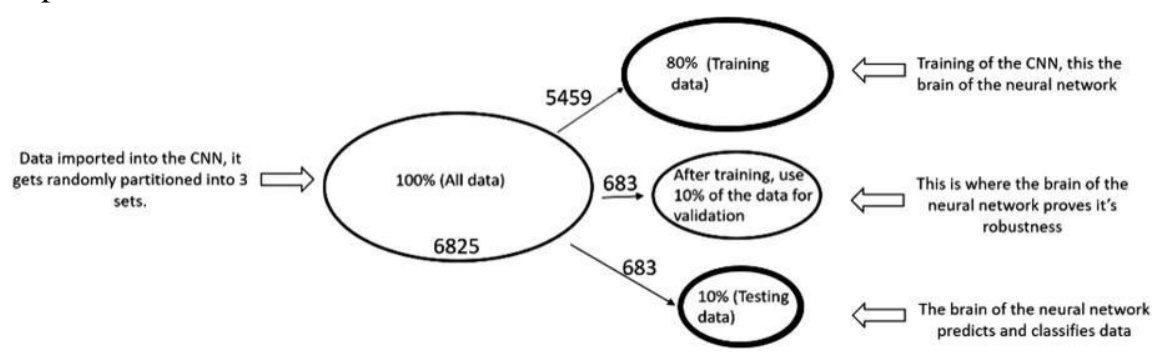

Fig. 4. Data pre-processing at rations of $80 \%$ learning, $10 \%$ validation and $10 \%$ testing from the $100 \%$ full dataset.

\section{Results and Discussion}

As previously hypothesized, hyperspectral images do indeed amplify rock differences by means of spectral signatures as shown by Fig. 5. This spectral technology combined with CNNs form a great tool from which future mining activities could be based due to their robustness.

\subsection{Hyperspectral Signatures}

From the 8 igneous rocks, the volcanic rocks (Fig. 5, lower row), 'rhyolite, andesite, basalt and dacite', which are rather difficult to distinguish in the field due to their fast cooling history, are easily distinguishable by visual observation of their hyperspectral
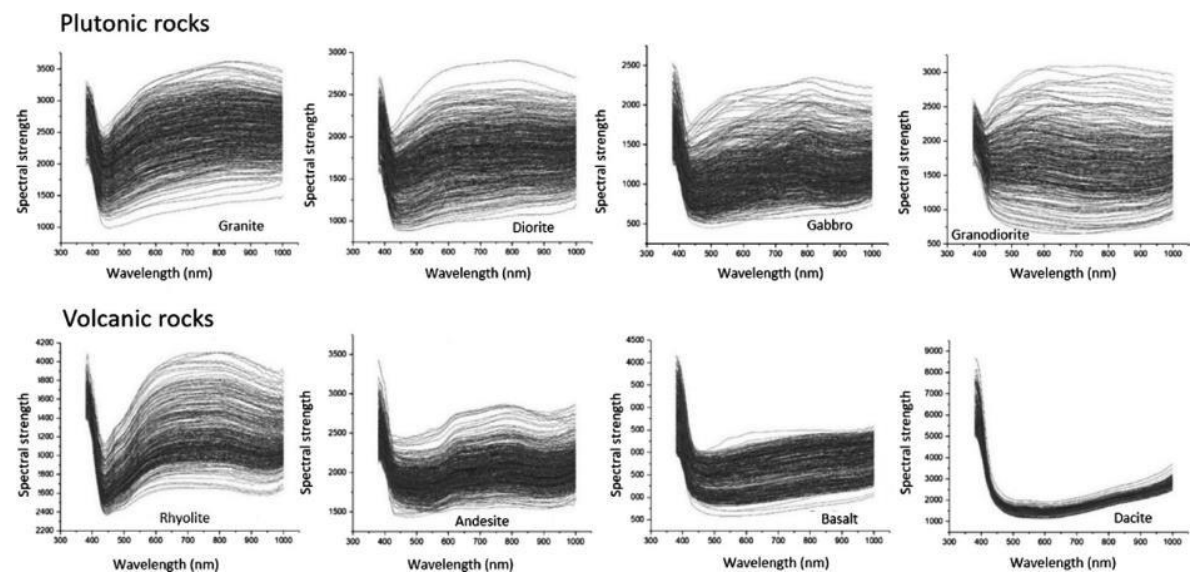

Fig. 5. Hyperspectral signatures of 8 plutonic and volcanic rock types, band shapes differ from rock to rock due to differences in interaction with light energy at given wavelengths.

signature band tilt, strength and curvature. However, their plutonic equivalents (Fig. 5, upper row), 'granite, diorite, gabbro and granodiorite', have different but slightly similar spectral signatures, hence the need to employ computer vision to thoroughly distinguish rocks with the option of database expansion.

\subsection{Deep Learning Analysis}

Our input, holds raw pixel values of each spectral image with a depth representing the entire VNIR. Simply, a CNN such as one in Fig. 6, consists of an input layer where data is fed, multiple hidden layers where the brain of the network is located, and an output layer where results are delivered. The network does this classification across all 125 layered bands since our input data contains 125 layers from the VNIR. 


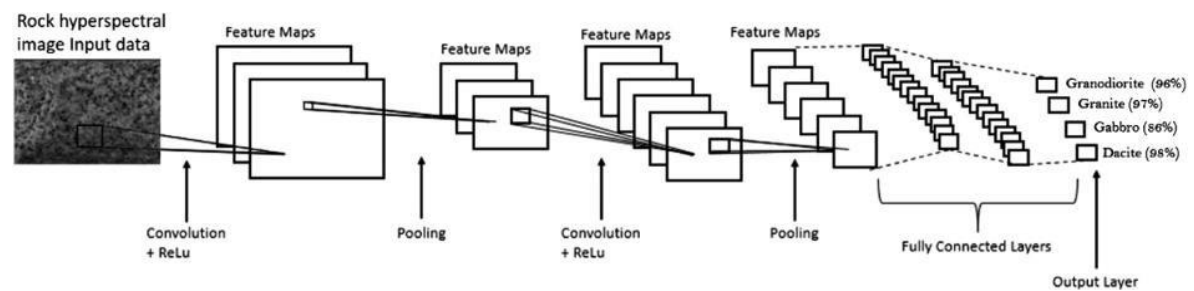

Fig. 6. Simplified architecture of a CNN with hyperspectral images as input, and rock classification as output.

The network presents 2 vital results after network training has been completed. The first, validation accuracy; communicates the recognition capabilities of the network at making predictions, the higher the validation output value, the more accurate the network is (Poria et al. 2016) at making predictions. The second vital variable is the test output result, this shows how capable the network is at running data which is unlabeled or whose origin is unknown against its database and predicting what that input data may most likely to be, based on already learnt data.

From Fig. 7A, the network shows a high validation accuracy of $96 \%$, this simply means the system is able to recognize each rock's spectral data imported into the system, suggesting that indeed deep learning has the ability to learn from multitudes of data fed into it, thereafter, predict the most probable answer centered upon the previously mentioned database, whilst simultaneously expanding its database.

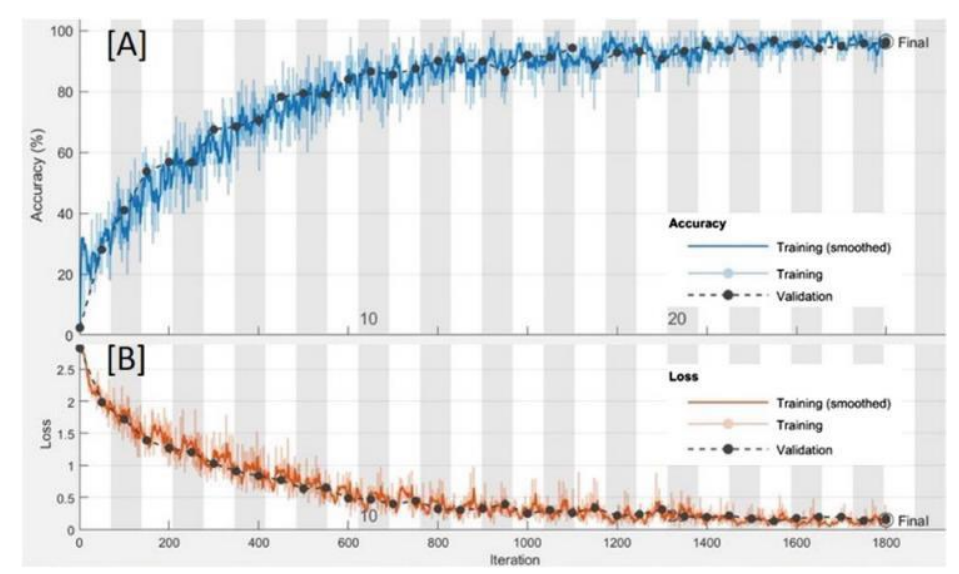

Fig. 7. CNN results. The network is highly capable of rock predictions as shown by the high validation percentage (96\%) during network training, and maintenance of a low prediction loss.

During training, the $\mathrm{CNN}$ is also able to show the relationship between the number of training steps/iterations, with training batches, also known as the prediction loss. The lower this value, the more accurate the CNN's prediction stability, also, the longer it preserves this low value, the more stable and reliable the network is (Xing et al. 2017) at predicting and classifying phenomena. Figure 7B shows how the network improves and maintains its prediction capabilities the more training steps employed; this variable, at time referred to as the epoch, is easily alterable to improve the network, it demonstrates how the number of iterations relate to prediction robustness accuracy.

Table 1 depicts all important information relating to the state of the $\mathrm{CNN}$, its prediction precision percentages and correctly recalled values from testing the rock characterization capabilities of the CNN. These results and their database can furthermore be applied to any rock engineering works which may need rock classification, database expansion is also one of the great advantages of deep learning as more data can be fed at any particular time to make predictions even more accurate and precise.

Table 1. CNN output results showing the test output predictions of each of the 8 igneous rock types.

\begin{tabular}{l|l|l|l|l|}
\hline Plutonic rock CNN results & \multicolumn{3}{l}{} \\
\hline Rock type & Granite & Diorite & Gabbro & Granodiorite \\
\hline
\end{tabular}




\begin{tabular}{l|l|l|l|l}
\hline Recalls/wrong scores & $99 / 3$ & $84 / 18$ & $88 / 14$ & $98 / 4$ \\
\hline CNN precision percentage (\%) & 97 & 82 & 86 & 96 \\
\hline
\end{tabular}

\begin{tabular}{l|l|l|l|l}
\hline Volcanic rock CNN results & Rhyolite & Andesite & Basalt & Dacite \\
\hline Rock type & $102 / 0$ & $149 / 5$ & $100 / 2$ & $100 / 2$ \\
\hline Recalls/wrong scores & 100 & 97 & 98 & 98 \\
\hline CNN precision percentages (\%) & &
\end{tabular}

\section{Conclusion}

From the presented proposal, it has been observed that hyperspectral imaging of rocks is highly capable of distinguishing rocks due to its high spectral resolution. Differences amongst rocks which the human eye is unable to observe are easily amplified by the use and application of hyperspectral imaging, making it easier for rock classification to take place in mining environments and beyond. This technology coupled with the deep learning $\mathrm{CNN}$, has succeeded in creating a database from which later predictions can be based. CNN results show a rock classification capability prediction accuracy of $96 \%$, hence this system is viable and has met above mentioned aims and objectives of this study by flawlessly classifying all 8 igneous rocks, hence worthy for consideration for mine planning and equipment selection.

\section{References}

Jia, F., Lein, Y., Lin, J., Zhou, X., Lu, N.: Deep neural networks: a promising tool for fault characteristic mining and intelligent diagnosis of rotating machinery with massive data. Mech. Syst. Signal Process. 72-73, 303-315 (2016)

Xing, J., Li, K., Hu, W., Yuan, C., Ling, H.: Diagnosing deep learning models for high accuracy age estimation from a single image. Pattern Recogn. 66, 106-116 (2017)

Simonyan, K., Vedaldi, A., Zisserman, A.: Deep inside convolutional networks: visualising image classification models and saliency maps (2013)

Kruse, F.A.: Mapping surface mineralogy using imaging spectrometry. Geomorphology 137, 41- 56 (2012)

Meer, F.: The effectiveness of spectral similarity measures for the analysis of hyperspectral imagery. Int. J. Appl. Earth Obs. Geoinf. 8, 3-17 (2006)

Pieters, C.M., et al.: The Moon Mineralogy Mapper (M3) on Chandrayaan-1. Curr. Sci. 96(4), 500-505 (2009)

Poria, S., Cambria, E., Gelbukh, A.: Aspect extraction for opinion mining with a deep convolutional neural network. Knowl.Based Syst. 108, 42-49 (2016)

Tompkins, S., Pieters, C.M.: Mineralogy of the lunar crust: results from Clementine. Meteor. Planet. Sci. 34, 25-41 (1999) 